Mario Grizelj

Ludwig-Maximilians-Universität München, mario.grizelj@germanistik.uni-muenchen.de

\section{Die Auferweckung des Lazarus}

Die Anhermeneutik des Ohrs, der singende Jesus und der Chor bei Johann Gottfried Herder

\section{Die Entwunderung im 18. Jahrhundert und Herders uneindeutige Wundertheologie}

Wunder sind zweifelhafte, außergewöhnliche Ereignisse. Sie versetzen in Staunen, faszinieren oder schrecken ab. Als staunenswert Unmögliches und Unerwartetes unter (meist) transzendenten Vorzeichen brechen sie in den Alltag und die Lebenswelt ein. ${ }^{1}$ Ihr epistemologischer und ontologischer Status ist unsicher und stark erklärungsbedürftig. Für die Aufklärung im Allgemeinen und die aufklärerische Theologie im Besonderen markieren sie in der Regel randständige Phänomene, die dem Aberglauben

1 Im Lateinischen, Englischen und Französischen kann man begrifflich differenzieren, indem man zwischen smirabilia (Natur-/Immanenzwunder, profan, relativ) und `miracula> (Transzendenzwunder, sakral, absolut) (vgl. hierzu im Rekurs auf Thomas von Aquin: Matuschek: Über das Staunen, S. 70 sowie Geppert/Kössler: Einleitung) sowie zwischen `wonderı, `marvel und `merveilleux ` (ein ungewöhnliches Ereignis) und `miracle (ein ungewöhnliches, religiöses Ereignis mit Bezug auf einen transzendenten Gott bzw. eine irgendwie geartete transzendente Macht) unterscheidet. Im Deutschen hat sich eine vergleichbare Unterscheidung (Wunder/Mirakel) nicht durchsetzen können (für eine ausführliche begriffliche Erörterung des >Wunders` siehe Grizelj: Wunder und Wunden).
Der Beitrag beleuchtet in einem ersten Schritt die Stellung Herders im Geflecht einer ,WunderAufklärungstheologie، (Offenbarung, stheologia naturalisı, Rationalismus, Supranaturalismus, Deismus, Neologie, Materialismus, historisch-kritische Bibelwissenschaft). In einem zweiten Schritt soll geklärt werden, in welchem Verhältnis Musikästhetik und Theologie stehen. Dabei ergibt sich erstens, dass die Botschaft der Bibel nicht über propositionales Wissen vermittelbar ist, sondern über die begeistert-emphatisch anhermeneutische Erfahrung der Erfahrung, und dass es zweitens vor allem die Musik ist, die diese Erfahrbarkeit der ambivalenten Erfahrbarkeit von Offenbarung und Wunder ausagiert. 
einer nicht mehr aktuellen, antimodernen Religiosität oder der Täuschung zugeschrieben werden. Im Rahmen der Säkularisierung müssten Wunder im Zuge des »linearen Religionsverfalls in Form einer zunehmenden Entkirchlichung, Privatisierung religiöser Einstellungen sowie einer Rationalisierung religiöser Weltbilder « keine strukturbildende Rolle mehr spielen; ${ }^{2}$ sie könnten höchstens als historische Marken fungieren, um vergangene Zeiten zu beschreiben. Wenn man jedoch die Säkularisierungsthese hinterfragt und annimmt, dass die Moderne zwar "viele überkommene Wunderwelten effektiv entzauberte oder zumindest deren Attraktivität minderte«, dass sie jedoch auch neue, ihrer Modernität geschuldete Wunderwelten schuf sowie verschiedenste, miteinander konkurrierende Modi der Wunderbeobachtung etablierte, dann öffnet sich der Forschung der Weg, den, wie es heißt, »forschungshemmenden Begriffsgegensatz von Ent- und Wiederverzauberung zu überwinden «, damit der sich "wandelnde[] Umgang mit wunderhaften Begebenheiten und wundersamem Handeln innerhalb der zugleich religiösen und säkularen Denk- und Wissenssysteme « der Moderne in den Blick gerät. ${ }^{3}$

Wie noch zu sehen sein wird, können Herders und Niemeyers Überlegungen genau dazu dienen, die Ambivalenz der Gleichzeitigkeit von religiösen und säkularen Denkmustern, die Gleichzeitigkeit von Ver- und Entzauberung zu veranschaulichen. Vor allem Herders Einstellung zum Wunder ist ambivalent, was jedoch nicht auf eine Inkonsistenz seines Denkens verweist, sondern als ein strukturelles Argument seiner theologischen, ästhetischen und poetologischen Modelle aufzufassen ist. Das einfache Ablösungsmodell, demzufolge das aufgeklärte 18. Jahrhundert und die aufgeklärte Moderne Wunder und Wunderglauben durch Rationalität ersetzen, muss mit Herder (und Niemeyer) hinterfragt werden. Ganz in diesem Sinne kann Daniel Weidner im Hinblick auf die Aufklärung zeigen, dass das »Wunder [...] nicht nur zentral für das Wissen der Aufklärung, sondern nicht weniger wichtig für das Wissen von der Aufklärung « ist. ${ }^{4}$

Die Wundertheologie des 18. Jahrhunderts, hier ist natürlich vor allem die protestantische gemeint, war lange Zeit vor allem eine Erklärungstheologie. Das Unerklärlich-Übersinnlich-Mirakulöse wurde wegrationalisiert, indem es überhaupt erklärt wurde. Dabei war es genau das UnerklärlichÜbersinnliche des Wunders, das eine rationalistische Theologie vor große Probleme stellen musste. ${ }^{5}$ So setzten auch verschiedene und freilich allesamt

2 Geppert/Kössler: Einleitung, S. 18.

3 Ebd., S. 15. Gegen die Dichotomie von »Fortschritt versus Entzauberung « wendet sich auch Gess: Staunen als ästhetische Emotion, S. 129.

4 Weidner: Un-Möglichkeit der Ausnahme, S. 123.

5 Vgl. hierzu Weidner: Un-Möglichkeit der Ausnahme und ders.: Lesen, Schreiben, Denken, S. 191. 
protestantische Versuche an, das Mirakulöse am Wunder wegzuerklären. Zu nennen wären hier vor allem i) die >rationalistische Wundererklärung`, die das Wunder als Wunderbares weitestgehend negiert, ${ }^{6}$ und ii) die sogenannte >Akkommodationstheorie von Johann Salomo Semler ( $†$ 1791), einem evangelischen Mitbegründer der historisch-kritischen Bibelwissenschaft, die davon ausgeht, dass sich Jesus grundsätzlich und auch im Hinblick auf seine Wundertaten an die Erwartungen und das Wirklichkeitsmodell seiner Zeitgenossen angepasst hat. Es geht also um eine Akkommodation an eine zeitgenössische Wunderfrömmigkeit, um »die ewigen Wahrheiten verständlich auszudrücken « ${ }^{7}$. Semler schreibt, »daß Jesus sich nach der sehr verschiedenen Fähigkeit derer gerichtet hat, in seinem Vortrage und Maas des Unterrichts, welche ihm jedesmal zuhörten «. ${ }^{8}$

Ebenfalls protestantisch geprägt ist iii) die Entmythologisierungstendenz. ${ }^{9}$ Hier wird davon ausgegangen, dass das Neue Testament aus einem mythologisch-mythischen Weltbild heraus geschrieben wurde, das inzwischen von einem wissenschaftlichen Weltbild abgelöst worden sei. Die Grundidee ist, den historisch-zeitgenössischen Jesus vom Christus des Glaubens, den predigenden und heilenden Jesus von Nazareth vom kerygmatischen Christus zu unterschieden, damit Kreuz und Auferstehung als Wunder Gottes und nicht Totenerweckungen oder Heilungen relevant werden. ${ }^{10}$

All diese Ansätze haben eines gemeinsam: Sie erklären am Unerklärlichen das Unerklärliche weg, domestizieren rational die Außergewöhnlichkeit und Unmöglichkeit des Wunders. Zimmermann spricht pointiert von einer "Ent-Wunderung « im Rahmen solcher Wundertextexegesen. ${ }^{11}$

6 Vgl. prominent Carl Friedrich Bahrdt (1741-1792), Carl Heinrich Venturini (1768-1849), Johann David Michaelis (1717-1791) und Heinrich Eberhard Gottlob Paulus (1761-1851), der für seine rationalistischen Wundererklärungen berüchtigt war (siehe Paulus: Philologischkritischer und historischer Commentar über die drey ersten Evangelien (1800-1802); vgl. hierzu Alkier: Wunder und Wirklichkeit in den Briefen des Apostels Paulus, S. 27 und insgesamt zu Tendenzen der protestantischen Wundertheologie seit der Aufklärung die komprimierten, aber ausgezeichneten Ausführungen in ders.: Jenseits von Entmythologisierung und Rehistorisierung sowie ders.: Wen wundert was? S. auch Weidner: Un-Möglichkeit der Ausnahme und Grizelj: Wunder und Wunden).

7 Alkier: Jenseits von Entmythologisierung und Rehistorisierung, S. 33. Zur Akkommodationstheorie siehe auch Zimmermann: Grundfragen zu den frühchristlichen Wundererzählungen, S. 10.

8 Semler: Abhandlung von freier Untersuchung des Canon, S. 25.

9 Johann Gottfried Eichhorn (1752-1827), Johann Philipp Gabler (1753-1826), David Friedrich Strauß (1808-1874) und später am berühmtesten Rudolf Bultmann (1884-1976) waren ihre Hauptvertreter.

10 Vgl. Zimmermann: Wundern über ’des Glaubens liebstes Kind , S. 107.

11 Zimmermann: Grundfragen zu den frühchristlichen Wundererzählungen, S. 12. - Auf der anderen Seite verweist Weidner auf den von Robert M. Burns so benannten »moderate empiricism« als »Hauptströmung der Verteidigung der Wunder im 18. Jahrhundert [...]: Sie will die 
Gemeint sind hier der Rationalismus und der, wie ich es nennen möchte, $>$ Botschaftismus, d. h. die Betonung auf die reigentlicheく Botschaft der Schrift, die die Grenzen sprengende, Wissensordnungen hinterfragende und Umformatierungen einleitende Dimension des mirakulösen Wunders nivelliert. Ruben Zimmermann fasst zusammen:

Statt die dem Wunder konstitutiv anhaftende Durchbrechung der Ordnung des Wissens anzuerkennen, wurde der Versuch unternommen, die Wundererzählungen in die bestehenden und sich ausweitenden Wissenssysteme einzupassen. [...] Die [Wunder-]Erzählung diente jeweils als Vehikel für eine übergeordnete Botschaft, die aber keineswegs an das Wunderhafte selbst gebunden ist. ${ }^{12}$

Dem liegt die medien- und literaturwissenschaftlich höchst problematische und epistemologisch als idealistisch zu klassifizierende Auffassung zugrunde, dass sich Ideen und Gedanken von ihren Ausdrucksformen trennen lassen, so wie »zwei chemische Substanzen«, formuliert es Stefan Alkier und konstatiert: »Das `Kerygma soll von seiner mythischen, >mirakulösen`Form fein säuberlich getrennt werden «, d. h.: »Der konkrete Wundertext gilt als uneigentlicher Träger einer >eigentlichen Botschaft $«{ }^{13}$ Unter anderem gegen diese Trennung schreibt Herder nicht nur als Philologe, sondern auch als Theologe an.

Der protestantische Pädagoge, Philologe, Literaturkritiker, Philosoph und Theologe Johann Gottfried Herder war mit seiner Auffassung von Wundern ein Problem für die protestantische Theologie. Dies vor allem aus zweierlei Hinsicht. Erstens ließ er sich nicht klar einer protestantischen Theologierichtung zuschreiben. Er war also kein Deist, kein Neologe, kein Pietist, kein moderater Empiriker, kein orthodoxer Lutheraner, kein Supranaturalist. ${ }^{14}$ Er nahm aufgrund dessen in vielen theologischen Fragen eine Gegensätze vermittelnde Mittelposition ein. Zweitens argumentiert er, wenn er als Theologe argumentiert, auch immer als Philologe und Poetologe. Seine Theologie lässt sich nicht von seiner Poetologie, Ästhetik

biblischen Wunder nicht im strengen Sinne beweisen, aber doch wahrscheinlich machen, dass sie, juristisch gesprochen, `beyond reasonable doubt` wahr sind « (Weidner: Un-Möglichkeit der Ausnahme, S. 132; Zitat im Zitat: Burns: The Great Debate on Miracles). Wir bewegen uns bei dieser Wunderverteidigung freilich auf protestantischem Boden.

12 Zimmermann: Wundern über `des Glaubens liebstes Kind،, S. 118 u. 119.

13 Alkier: Jenseits von Entmythologisierung und Rehistorisierung, S. 41 u. 40. Analog hierzu beobachtet Weidner die »Frage nach literalem und allegorischem Verständnis« als ein entscheidendes »hermeutisch-philologische[s] Problem der Wunderdebatte im 18. Jahrhundert « (Weidner: Un-Möglichkeit der Ausnahme, S. 129).

14 Dass für Herder die deutsche Version eines stark gemäßigten Deismus, die Neologie, keine Option war, lässt sich in den Provinzialblättern (1774) und vor allem in seiner heftigen SpaldingKritik nachlesen. 
und Philologie trennen. Nicht umsonst kann es dann heißen: »Insofern versucht Herder in seinen exegetischen Arbeiten zwischen wundergläubigem Supranaturalismus und kritischem Rationalismus hindurchzusteuern und einen vermittlungstheologischen Weg einzuschlagen. $\aleph^{15}$ Er manövriert zwischen der Skylla der rationalistischen Bibelkritik und der Charybdis der konservativen Verteidiger der Heiligkeit der Bibel. Für Herder ist die Bibel beides: Heilige Schrift und Heilige Schrift.

Bei Herder kann von einer "anthropologischen Religionstheologie» gesprochen werden. ${ }^{16}$ Herder stellt die »anthropologische Maxime« auf, wonach »die biblischen Schriften als menschliche, das heißt als geschichtlichliterarische Urkunden zu lesen « sind. ${ }^{17}$ Dementsprechend ist eines seiner theologischen Hauptargumente die Verbindung von historisch kontextualisierender Deutung der Bibel und Anerkennung der `literarischen` Form derselben. Dabei geht er weder davon aus, dass es einen literalen Sinn der biblischen Ereignisse, hier konkret der Wunder gibt, noch dass sich eine allegorische Deutung sauber von ihrem Schriftsinn ablösen ließe (es gibt

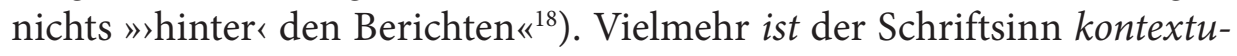
ell der allegorische Sinn und vice versa - und zwar sowohl für die in der Textontologie Anwesenden (= >discours` und `histoire $\triangleleft$ ) als auch für die Adressaten (= Rezeption) des Berichts: »Wie sie [die Verklärung] bewirkt sei, kann und werde ich nicht erklären; ich erläutere sie als Begebenheit dieser Geschichte, was sie den Umstehenden [= >histoire`] war und in der Erzählung [ = >discours und Rezeption] bedeuten sollte $« .{ }^{19}$ Damit verlagert er das Wunderproblem von der Sachebene auf die Darstellungsebene. Die Möglichkeit von Wundern ist keine Frage der Ontologie oder der Metaphysik und auch keine der Epistemologie, sondern eine Frage der Darstellung, konkret der Narration. ${ }^{20}$

$\mathrm{Zu}$ dieser Betonung des Narrativen kommt ein wichtiger Wunderaspekt hinzu. Herder interessiert sich im Rahmen seiner anthropologischen Religi-

15 Bultmann/Buntfuß/Cordemann/Keßler: II.2 Theologie, S. 348.

16 Ebd., S. 339. Die Frage, ob Herder von Jesus im humanistischen Sinne als einem Menschensohn ausgeht (vgl. Arnold: Von den letzten Dingen; s. hierzu bspw. »daß Christus ein Mensch wie wir gewesen « [Herder: Theologische Briefe, S. 497]) oder ein christologisches Jesusbild liefert (vgl. Cordemann: Herders christlicher Monismus), wird kontrovers diskutiert.

17 Bultmann/Buntfuß/Cordemann/Keßler: II.2 Theologie, S. 344.

18 Weidner: Lesen, Schreiben, Denken, S. 190. Weidner spricht in diesem Kontext auch von »Herders Unklarheit in der Wunderfrage" (ebd.).

19 Herder: Vom Erlöser der Menschen, S. 654 (meine Hinzufügungen in eckigen Klammern) (vgl. hierzu auch Weidner: Lesen, Schreiben, Denken, S. 189).

20 Genauso verfährt auch, ohne sich allerdings explizit auf Herder zu beziehen, das groß angelegte von Zimmermann herausgegebene Wunderkompendium. 
onstheologie signifikanterweise für Aspekte, die das Anthropologische übersteigen. Er liest einzelne Bibelstellen als Momente von »inkommensurable[r] Bedeutung $«{ }^{21}$ Hierbei dient ihm das Wunder als Exemplum, um die Kombination von Wissen und Inkommensurabilität zu bewerkstelligen und dabei auch das Verhältnis von Anthropologie (nur Mensch) und Sakralität (der Mensch als Heiliger) auszutarieren. Dabei geht Herder davon aus, wie wir schon sehen konnten, dass gerade die Wunder weder strikt rationalisierbar sind noch einfach als Evidenzen des Wunderbaren zu lesen seien. Es ist diese unhintergehbare Ambivalenz der Wunder, die ihren ontologischen und epistemologischen Status bestimmt und die Wunder als Wahrheiten der Wundererzählungen ausweist. Dieses Argument entwickelt Herder in seiner Auseinandersetzung mit Hermann Samuel Reimarus:

Herder kehrt die Argumentationslogik gegenüber Reimarus um. Gerade die von Reimarus konstatierten Widersprüche zwischen neutestamentlichen Berichten von der Auferstehung sprächen für die Glaubwürdigkeit der Auferstehungszeugen. Eine einheitliche, widerspruchsfreie Darstellung wäre ein wesentlich höheres Indiz für einen verabredeten Betrug der Jünger. ${ }^{22}$

Die Wundererzählungen bezeugen gerade aufgrund ihres ambivalenten Darstellungsmodus die Wahrheit der leibhaftigen Auferstehung für die Jünger und für die zeitgenössischen Rezipienten. D.h., die Jünger sind dem leibhaftig Auferstandenen realiter begegnet und dies haben die zeitgenössischen Rezipienten geglaubt. Gleichwohl geht es nicht darum, dass Jesus materialiter auferstanden ist, da seine Leibhaftigkeit keinen körperlichen, sondern einen intelligiblen Sinn buchstäblich verkörpert: »Die Auferstehung Christi war eine Wiedergeburt der Apostel zu neuen Ideen und Hoffnungen, zu einer Wirksamkeit bis an ihr Lebensende. $«^{23}$ So lässt sich eine unauflösbare Spannung zwischen literalem auf der einen und idealem bzw. allegorischem bzw. kerygmatischem Sinn der Wunderberichte auf der anderen Seite ausmachen. Es ist gerade diese Spannung, in der sich sowohl die Uneindeutigkeit des Wunders (Objektebene) als auch die Uneindeutigkeit einer Wundertheologie (Beobachtungsebene) äußert. Diese beiden Uneindeutigkeiten sind produktiv und verweigern sich einer deistischen und neologischen Domestizierung des Wunders, ebenso wie sie sich einer antiaufklärerischen Supranaturalisierung entgegenstellen. Und sie sind nicht nur argumentationslogisch produktiv, sie sind vielmehr Ausdruck der In- 
kommensurabilität der göttlichen Botschaft. Gott kommuniziert im Modus des Uneindeutigen mit den Menschen und die Wunder sind exemplarische Formen dieser uneindeutigen Kommunikation. ${ }^{24}$

Aus Herders Forcierung von Spannung und Ambivalenz zuungunsten klarer Verhältnisse (seien sie deistischer oder supranaturalistischer Natur) lassen sich dann (mindestens) zwei Konsequenzen ableiten: i) Das Aushalten der Ambivalenz ist eine genuin religiöse Erfahrung, die theologisch erfasst werden kann, da das Ungeheuerliche der Offenbarung nur im Modus des Uneindeutigen erkannt wird und ii) dieses theologische Argument ist schon Teil einer Säkularisierungstendenz, da religiöse Erfahrung allein im Modus des Ästhetischen zu einer ambivalenten Erfahrung geworden ist. Das auf Ambivalenz ausgerichtete theologische Argument wäre solchermaßen Epiphänomen von Säkularisierungstendenzen im letzten Drittel des 18. Jahrhunderts, die die religiöse Erfahrung nur ästhetisch vermittelt betrachten können. Religiöse Erfahrung wäre hier eben nicht mehr genuin religiös, sondern schon ästhetisch säkularisiert. Zudem spräche Herder hier als Philologe und Ästhetiker über religiöse Fragen und nicht mehr als Theologe von religiöser Erfahrung.

\section{Die Anhermeneutik des Ohrs}

Herder argumentiert nicht nur, dass das Wunder im Modus der Ambivalenz erfahren wird, bzw. dass im Wunder Ambivalenz erfahren wird, sondern

24 Für eine bestechende Analyse der Korrelation von Wunder und Wundernarration siehe Welcks Johannesexegese. Welck kann u.a. zeigen, dass das Johannesevangelium formale und narratologische Brüche und Inkonsistenzen gerade in seinem Umgang mit Wundern aufweist. Das Erzählen von Wundern (’histoire`) schlägt sich auf den Modus des Erzählens (`discours`) nieder, durch »Einschübe, Akzentuierungen und Nachträge wird [...] die Darstellung des Wunders in seiner Geradlinigkeit beeinträchtigt, die Einsträngigkeit aufgehoben« (Welck: Erzählte Zeichen, S. 62f.). Für Welck ergibt sich hieraus eine theologische Produktivität von Ambivalenz, Störung und Gebrochenheit, die es den Lesenden »erlaubt, das Störende als Element eines neuartigen, eigentümlichen literarischen Zusammenhangs jenseits des erwarteten zu erkennen, durch welchen ihm ein neuartiges, eigentümliches Handeln Gottes gegenüber den Menschen vor Augen geführt wird « (ebd., S. 253). Vgl. hierzu auch überzeugend Zimmermann: Grundfragen zu den frühchristlichen Wundererzählungen, S. 15: »Was hier erzählt wird, soll nicht religionsgeschichtlich angepasst, rational plausibilisiert oder bildlich relativiert werden. Es soll Furcht und Schrecken auslösen, beim Lesenden selbst Irritationen und Fragen hervorrufen [...]. Es soll gerade Bekanntes, Rationales und Plausibles in Frage gestellt werden. Diese Verunsicherung und Furcht darf keineswegs heruntergespielt oder exegetisch gefügig gemacht werden. Sie ist aber auch keine Furcht, die lähmt oder verzweifeln lässt. Sie ist produktiv und wirksam und führt letztlich zur Erkenntnis. Sie befördert eine `Heuristik der Furcht«". - Dass Wundergeschichten "Irritationen, Abweichungen« und »Brüche in den Text [Neues Testament] « einschreiben, ist das Argument von Alkier: Jenseits von Entmythologisierung und Rehistorisierung, S. 30, vgl. auch S. 38. 
auch, dass Ambivalenz nicht rationalistisch, sondern vielmehr im Modus der Begeisterung zu erfahren ist: „Der ideale Leser der Offenbarung ist der begeisterte Liebhaber ${ }^{25}$ Statt sturer Schriftexegese wird hier einer emphatischen Begeisterung vertraut. Dabei korrelieren die Ambivalenz des Wunders und die Begeisterung, was nicht nur für die religiöse Erfahrung gilt, sondern auch für die Theologie, denn: »Philologie ohne Empathie bleibe blind für den religiösen Gehalt der biblischen Schriften $«{ }^{26}$ Die Besonderheit und Exorbitanz der Offenbarung und konkret der Wunder lässt sich nicht einfach verstandesmäßig erfassen. Die Hermeneutik kommt hier an ihre Grenzen. Um die Bibel, die Offenbarung und die Wunder zu verstehen - es geht bei Herder weiterhin um das Verstehen und nicht um reines Erleben - muss mit der Begeisterung ein anhermeneutischer Modus gefunden werden. ${ }^{27}$ Dabei geht es eben nicht um die Erfassung der Bedeutung als Botschaft, sondern um die Erfahrbarkeit der ambivalenten Erfahrbarkeit von Offenbarung und Wunder. Die Botschaft der Bibel ist nicht über proportionales Wissen vermittelbar, sondern über die begeistert-emphatisch anhermeneutische Erfahrung der Erfahrung. ${ }^{28}$ Die Botschaft der Offenbarung und der Wunder ist keine reine Botschaft, keine Kommunikabilie, die hermeneutisch isoliert und detektiert werden kann, sondern sie äußert sich im uneindeutigen Kommunikationsmodus und der dadurch evozierten uneindeutigen Erfahrung. Der ambivalente Kommunikationsmodus is the message, ließe sich salopp formulieren.

Meine gesamte Argumentation zielt nun auf den zentralen Punkt ab, die bisher entfaltete uneindeutige Kommunikation mit Herders Ansichten zur Akustik und Musik zusammenzuführen. Es ist überraschend, dass es bisher noch keinen diesbezüglichen Versuch in der Forschung gibt. Die Forschung hat bisher die beiden Bereiche Theologie und Musik (Akustik) weitestgehend getrennt analysiert. Meine These lautet indes: In der Akustik und der Musik finden wir genau den Modus von uneindeutiger Erfahrung, der mit der Uneindeutigkeit der Wunder und der Wundererzählungen korreliert. ${ }^{29}$

Bultmann/Buntfuß/Cordemann/Keßler: II.2 Theologie, S. 350.

Ebd., S. 353.

Den Begriff des >Anhermeneutischen`, der indiziert, dass es Texte und Artefakte gibt, die nicht »nach den Regeln der Hermeneutik zu entzifferen, zu lesen und auszulegen « wären, habe ich von Teuber übernommen (Teuber: Sichtbare Wundmale und unsichtbare Durchbohrung, S. 171).

28 Buntfuß argumentiert ähnlich und spricht, konkret im Hinblick auf Herders Deutung der Apokalypse, von einer "performativen Deutung der Apokalypse« (ebd.).

9 In diesem Beitrag kann die wichtige Unterscheidung zwischen Musik und Akustik nicht erörtert werden (s. hierzu überzeugend Schneider: Ins Ohr geschrieben, S. 113-127 (in Bezug auf Herder) und S. 9-20 (allgemein)). 
Joh. Nikolaus Schneider kann zeigen, dass Herder im Umgang mit der Schrift (im Allgemeinen, also inkl. Literatur, Bibel und aller anderen Texte) darauf ausgerichtet ist, »Lese- und Interpretationstechniken zu entwickeln, die es ermöglichen - trotz Schriftlichkeit - die stummen Schriftzeichen zu verlebendigen ${ }^{30}{ }^{30}$ In diesem Zusammenhang und in Auseinandersetzung mit Baumgartens Aesthetica argumentiert Herder wahrnehmungstheoretisch, indem er das Gehör zwischen dem Sehsinn (Distanz, Kälte, Künstlichkeit, philosophische Erkenntnis) und dem Tastsinn als »die unmittelbarste aber deshalb auch undifferenzierteste Wahrnehmungsart " platziert: ${ }^{31}$

Das Gehör allein ist der innigste, der tiefste der Sinne. Nicht so deutlich wie das Auge, ist es auch nicht so kalt; nicht so gründlich wie das Gefühl [= Tastsinn], ist es auch nicht so grob; aber es ist so der Empfindung am nächsten wie das Auge den Ideen und das Gefühl der Einbildungskraft. Die Natur selbst hat diese Nahheit bestätigt, da sie keinen Weg zur Seele besser wußte als durch Ohr und - Sprache. ${ }^{32}$

Das Ohr ist indes nicht nur zwischen dem Seh- und Tastsinn angeordnet, es muss vielmehr auch die Spannung dieser Mittelposition aushalten und ist solchermaßen der ambivalente Sinn, jener Sinn, der weder kalt und distanziert noch unmittelbar ist, der Sinn, der sowohl ein wenig kalt und distanziert als auch ein wenig direkt ist. Das Ohr als Sinnesorgan liegt zwischen Verstand (sehen) und Einbildungskraft (tasten) und konvergiert mit der Empfindung. Als solches vermittelt es zwischen den Polen, ${ }^{33}$ muss aber auch die Distanz zwischen Verstand und Einbildungskraft aufrechterhalten, um seine Vermittlungsposition erhalten zu können.

Die These wäre nun, dass die Ambivalenz der Wundererzählungen mit dieser spannungsvollen Mittlerposition des Gehörsinns korreliert. Die Wundererzählungen sollen in ihrer exorbitanten Wirkung weder (nur) den Verstand noch (nur) die Einbildungskraft affizieren - im ersten Fall müssten sie rational wegerklärt werden (im Sinne des Deismus, der historischkritischen Bibelkritik und der Neologie), im zweiten müssten die Wunder nur bestaunt werden im Sinne eines >ekplettein` (was mit einem - neoplatonischen - Supernaturalismus konvergieren würde). ${ }^{34}$ Die Wunder agieren

Ebd., S. 114. Die folgende Argumentation stützt sich im Wesentlichen auf Schneiders Ergebnisse.

Ebd., S. $116 f$.

32 Herder: Viertes Kritisches Wäldchen, S. 357.

33 Vgl. Schneider: Ins Ohr geschrieben, S. 125.

34 Wie Matuschek im Hinblick auf die aristotelische Metaphysik (erstes Buch) und Platons Theaitetos zeigen kann, lässt sich zwischen der Überwindung des Staunens durch den Wissensgewinn (>athaumastia< bei Aristoteles) und einer Steigerung des Staunens (>ekplettein bei Platon) unterscheiden (vgl. hierzu Matuschek: Über das Staunen, S. 20; Gess: Staunen als ästhetische Emotion, S. 121f.; Schawelka: Kunst und Wunder, S. 29 sowie Grizelj: Wunder und Wunden). 
dem Gehör entsprechend uneindeutig zwischen Wissen und `ekplettein « und können solchermaßen tief in den Menschen eindringen und so seine Seele erreichen. ${ }^{35}$ Das Gehör ist damit der Sinn der Tiefe, der Sinn, der Oberflächen durchdringen kann, während der Seh- und der Tastsinn an der Oberfläche haften bleiben. Es ist dabei signifikant, dass mit der Tiefe auch eine Uneindeutigkeit verknüpft ist: »Jene [die akustischen Wirkungen] würken gleichsam in einander, durch Schwingungen, die in Schwingungen fallen: sie sind also nicht so aus einander, nicht so deutlich «. ${ }^{36}$ Solchermaßen gehen sie » tiefer als das `Superfizielle der Abstraktion $« .{ }^{37}$ Damit befinden wir uns mit Herder - gemäß der vier Felder, die diesen Sammelband strukturieren -, sowohl auf dem Feld des `musikalischen Erschreibens` als auch auf dem Feld der `Topographien der Literatur «. Mit der genannten Tiefe ist auch das Dunkle und damit das Uneindeutige und Unklare korreliert. Und was für die Poesie gilt, dass sie nur als Uneindeutige ihre volle Wirkung entfalten kann, ${ }^{38}$ gilt, so meine These, ebenso für die Offenbarung und die Wundererzählungen. Das von der Forschung erarbeitete musikalische Dispositiv bei Herder - »Unbestimmtheit des Akustischen « ${ }^{39}$ musikalische Erfahrung vs. Rationalität und Unbestimmtheit des Rhythmischen ${ }^{40}$ - führt uns auf den Pfad des >Anhermeneutischen . Die Unbestimmtheit des Akustischen und der Musik und all der mit diesen verbundenen Phänomenen (Schall, Ton, Rhythmus usw.) eröffnet eine Dimension, in der die Erfahrung und das Verstehen über den Akt des Äußerns laufen zuungunsten des Geäußerten. Das, was für die mystische Kommunikation gilt, dass sie »keinen Inhalt [hat]; sie ist Äußerungsakt ohne Botschaft, pure énonciation ohne reales énoncé«, ${ }^{41}$ gilt auch für das Akustische und die Musik bei Herder. Wenn es über die Mystik heißt: »Es ist der Akt der énonciation, in dem nichts Bestimmtes ‘angekündigt ‘, oder >annonciert $\iota$, jedoch etwas Unnennbares

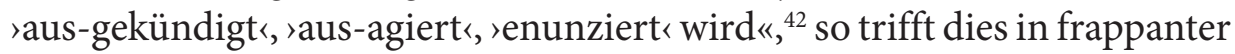

Vgl. hierzu Herder: Viertes Kritisches Wäldchen, S. 292 (zit. in Previšić: Akustische Paradigmen vor der Philologie: Herder, S. 341): »Die Würkungen dessen, was in unser Ohr einfließt, liegen gleichsam tiefer in unserer Seele, da die Gegenstände des Auges ruhig vor uns liegen.« Herder: Viertes Kritisches Wäldchen, S. 292 (zit. in ebd.). Ebd.

38 Siehe hierzu Köhler: Natur und Form, S. 27: Herder geht »über diese für seine Zeit ungewöhnliche Einschätzung der Musik noch hinaus, wenn er schließlich die ästhetische Norm umdreht, indem er behauptet, die Deutlichkeit sei ein ästhetisches Defizit sünstlerischer Wirkung « (zit. in Previšić: Akustische Paradigmen vor der Philologie: Herder, S. 342).

39 Ebd., S. 343.

40 Vgl. ebd., S. $344 f$.

41 Teuber: Die mystische Mär, S. 23.

42 Ebd., S. 24. 
Entsprechung auf Herders Konzeption des Akustischen und der Musik zu - und, so meine These, auch auf die Wirkungsdimension der Offenbarung und des Wunders. Das Wunder lebt, so legen es meine obigen Ausführungen nahe, von der spannungsgeladenen und ambivalenten Erfahrung der in der Wundernarration ausagierten >énonciation $`$ der Uneindeutigkeit des Wunders, wobei das rénoncér (das Wunder selbst) in den Hintergrund tritt. Wie die Musik über das Akustische vor allem ihre >énonciation > >auskündigt‘, so berichtet die Wundererzählung vor allem von ihrem Berichten selbst und den damit verbundenen Wirkungen. ${ }^{43}$

\section{Der singende Jesus auf der Bühne}

Herder hat die hier unterstellte Konvergenz von Wundertheologie und $\mathrm{Mu}$ sikästhetik nicht systematisch herausgestellt, er hat sie auch nicht explizit reflektiert. Dennoch erlauben es die Befunde, Theologie, Musikästhetik, Philologie, Poetologie und Ästhetik in der dargestellten Form miteinander zu korrelieren. Es gibt nun einen kleinen Text, dem Herder selbst nicht viel abgewinnen konnte und der von der Forschung im Grunde ignoriert wird: Die Rede ist vom Libretto Die Auferweckung des Lazarus. Eine biblische Geschichte zur Musik (1772), das im Inhaltsverzeichnis als Kantate bezeichnet wird. ${ }^{44}$ Herder liefert hier seine Version der Lazarusperikope des Johannesevangeliums (Joh 11,1-44), wobei er eine entscheidende Variation einbaut: Jesus erweckt den toten Lazarus, jedoch nicht, damit dieser wieder unter den Lebenden wandelt als Zeichen der Allmacht Gottes und als Vorzeichen der späteren Auferstehung Jesu, sondern damit Lazarus im Bruderkuss

43 Für eine hervorragende, sowohl theoretische als auch fallorientierte, Diskussion des Verhältnisses von Sprache und Musik s. die Beiträge in Berger: Musik jenseits der Grenze der Sprache; s. auch Lütteken: Das Monologische als Denkform in der Musik zwischen 1760 und 1785, S. 85-103. Hajduk spricht in diesem Zusammenhang von einer "physikalisch-psychologischen Doppelperspektive« Herders, da dieser »im Zusammenhang von Ton- und Literaturästhetik durchaus nach akustischen und seelischen Verhältnisbestimmungen sucht (Poetologie der Stimmung, S. 391).

44 Wohlgemerkt im Inhaltsverzeichnis der Sämtlichen Werke, Band XXXIII; im Band XXVIII derselben Ausgabe fehlt wiederum die Bezeichnung Kantate. - Heidrich weist darauf hin, dass im 18. Jahrhundert nicht klar zwischen Oratorium und Kantate unterschieden wird. Es gibt oft synonyme Verwendungsweisen. Der Versuch, für das Oratorium eine dramatisch-erzählende Struktur als notwendig anzunehmen, die Kantate jedoch nur als Zusammenstellung von »'Sprüchen, Arien und Gesängen« « zu beschreiben, setzt sich nicht flächendeckend durch, da durch ambivalente Aufführungspraxen nicht klar ist, welche Form gottesdienstlichen Anforderungen entspricht und welche nicht (Heidrich: Die protestantische Kirchenmusikanschauung im 18. Jahrhundert, S. 199; vgl. dort auch S. 184). 
mit seiner Schwester »im Todeskuß zum schönern Leben - / Gen Himmel hin, die schöne Bahn « fährt. ${ }^{45}$ Lazarus wird erweckt, um gleich wieder zu sterben, um in diesem Wechselspiel von Leben und Tod das ewige Leben bewusst zu erfahren und diese Erfahrung zu kommunizieren. ${ }^{46}$

Neben dieser einschneidenden Änderung fällt auf, dass das Libretto stark dialogorientiert ist. Die Betonung liegt auf den Stimmen und damit auf den Solisten. Dadurch erhält dieses Libretto opernhafte Züge. 'Story` und `plot $`$ werden ohne Erzählerstimme allein durch die Solisten getragen (Maria, Martha, Jesus, Lazarus) - besonders auffällig ist dies im längeren Duett zwischen Maria und Lazarus nach dessen Auferweckung. Gleichwohl gibt es, ganz in der protestantischen Oratoriumstradition, daneben auch Choräle, was insgesamt eine eigenwillige Kombination von Dialog und Choral bzw. opernhaften Zügen und protestantischen Oratoriumsstrukturen ergibt. Dies führt dann dazu, dass wir uns zwar eindeutig auf dem Feld des >musikalischen Erschreibens bewegen, denn es handelt sich um ein Libretto, das explizit durch das intendierte Singen die klanglichen Qualitäten des Dargestellten ausstellt und die >énonciation` in Form von klanglichen Figuren und Strukturen präsentiert; durch das Opernhaft-Dialoghafte kommt aber eine Dimension hinein, die diese biblische Geschichte nicht nur hörbar, sondern auch lesbar macht. Der gesungene Dialog ist darauf angelegt, nicht nur gesungen, sondern auch verstanden zu werden. Kurzum, die Frage ist: Welchen Stellenwert nimmt die Sachdimension, das Geäußerte (`énoncé ), in der Oper, welche im Oratorium und welche in Herders Hybrid ein?

In diesem Kontext ist besonders auffällig, dass Jesus eine Solostimme bekommt. Er tritt auf der Bühne auf und ist Teil der >dramatis personae Dies ist im 18. Jahrhundert keineswegs selbstverständlich. Friedrich Gottlieb Klopstock schrieb im Vorwort seines Bibeldramas Der Tod Adams (1753/57), dass er sich durchaus bewusst ist, dass es schwierig sei, "große[] Männer der Offenbarung « auf die Bühne zu bringen, da diese, »die nicht anders, als von den tiefsten Geheimnissen der Religion begleitet, aufgeführt werden könnten, selbst für das ernsthafte Trauerspiel zu ernsthaft sind «. ${ }^{47}$ Wenn man sie dennoch auf die Bühne bringt, so sei das legitim, da hier der »Vorhof zum Heiligthume« betreten wird..$^{48}$ In diesem Vorhof besitzt

Herder: Die Auferweckung des Lazarus, S. 517.

Die übliche Deutung, dass durch diese Änderung ein Trauerfall im Haus Schaumburg-Lippe verarbeitet wird, mag zwar kontextgeschichtlich stimmen, ist aber hermeneutisch äußerst unbefriedigend. Hinter der Änderung steckt auch theologisches und ästhetisches Kalkül.

Klopstock: Biblische Dramen, S. 5.

Ebd. 
das, was geschieht, eine "gewisse Miene von Weltlichkeit «. ${ }^{49}$ Hier ist eine gattungstheoretische Aussage mit einer grundsätzlich poetologischen und einer theologischen verbunden: Im Drama, auf der Bühne haben wir im Hinblick auf die Darstellung religiöser Stoffe ein hybrides Medium; es wird Geoffenbartes dargestellt, aber noch diesseits der Grenze von Immanenz und Transzendenz. Das Dargestellte bleibt, weil es eben auf der Bühne gezeigt und gespielt wird, noch weltlich-immanent verankert. Die beiden Unterscheidungen Stoff/Form und Immanenz/Transzendenz sind im Hinblick auf das Religiöse gattungstheoretisch miteinander verbunden, da es das religiöse Epos ist (sprich der Messias), der das Heiligtum schon betreten hat, damit die Weltlichkeit abgestreift hat, während das Drama, auch wenn es als Tragödie einen religiösen Stoff darstellt, noch im Vorhofe verweilt. »Klopstocks Entwurf einer `heiligen Poesie` [ist hier] gattungsmäßig differenziert « ${ }^{50}$ Die 'heilige Poesier meint den Messias, das Epos, und nicht das biblische Drama. Aber nicht nur Form/Stoff und Immanenz/Transzendenz sind miteinander korreliert, hinzu kommt noch die Unterscheidung von Altem und Neuem Testament. Alle drei Bibeldramen Klopstocks behandeln Figuren des Alten Testaments (Adam, Salomo, David), ${ }^{51}$ das heißt, dass die Darstellung des Alten Testaments dem Theater, dem das Weltliche noch anhaftet, vorbehalten ist, während das transzendent-christologische Moment dem Epos entspricht und die Poesie als `heilige Poesie von der Welt und dem Irdischen loslöst. Das Alte Testament und das Drama markieren den Vorhof zum Heiligtum, während das Epos und das Neue Testament es betreten haben. Hier liefert uns Klopstock eine christologische Poetologie, die in der Korrelation von Altem Testament und Drama poetologisch-ästhetische und theologische Aussagen ineinander verschränkt. Gleichwohl ist sich Klopstock der Schwierigkeiten dieser poetologisch-gattungstheoretischen Konstellation bewusst, weshalb er sein Vorwort zum Tod Adams mit dem Satz enden lässt, dass er »einsieht, daß sein Stück, wegen gewisser Nebenumstände, nicht aufs Theater gehöret $«{ }^{52}$ Entscheidend ist nun, dass Klopstock aus theologischen, gattungstheoretischen und gattungsgeschichtlichen Gründen niemals Jesus auf der Bühne gezeigt hätte, Jesus konnte allein im Epos sauftreten`.

Herder hingegen kann im Rahmen seiner anthropologischen Religionstheologie Jesus durchaus auf die Bühne bringen. Wenn Jesus als Mensch seine Göttlichkeit zeigt, dann kann er genauso reden und sprechen und 
genauso singen wie alle anderen Menschen. Jesus ist bei Herder bühnenreif und sogar soweit anthropomorphisiert, dass er Teil eines opernhaften Bühnensettings werden kann. Jesus ist Teil eines strukturell stark opernhaften, d.h. immanent-weltlichen geistlichen Oratoriums. Wiederum haben wir hier eine sehr enge Korrelation von Poetologie und Theologie.

August Hermann Niemeyer (1754-1828) hingegen verweigert Jesus den Bühnenauftritt. Niemeyer war stark neologisch beeinflusst (durch Johann Salomon Semler (1725-1791) und vor allem durch Johann August Nösselt (1734-1807)) und schrieb 1778 Lazarus. Religiöses Drama (Kantate, Oratorium) in 3 Akten, das dann 1820 von Franz Schubert vertont wurde (Lazarus, D 689). ${ }^{53}$ Aus theologischen und gattungstheoretischen Gründen möchte Niemeyer Jesus Christus nicht auf die Bühne bringen. Die Person Christi ist für die profane Bühne viel zu heilig, zu heilig sogar für das Oratorium..$^{54}$ Jesus ist nicht gleichermaßen darstellbar wie die anderen Figuren; wenn es die Bibelgeschichte aufnötigt, Jesus doch als Hauptfigur führen zu müssen, so wie in der johanneischen Lazarusperikope, so muss Jesus abwesend anwesend sein. Handlungen und Worte Jesu werden qua Botenbericht bzw. indirekter Rede und Zitat dargestellt:

Im religioesen Drama so viel man auf der einen Seite dadurch gewinnen koennte, glaub ich dies Recht [Jesus auf der Buehne durch einen Saenger darstellen zu lassen] dem Dichter voellig abschneiden zu muessen. Die Person des Erloesers ist viel zu heilig, als daß man sie von einem Saenger, unter denen man so wenig waehlen kann, und bey welchem die gute Stimme oft das einzige Gute ist, koennte vorstellen lassen. Selbst das Singen hat mir da eine Nebenidee, die leicht die Ehrerbietigkeit schwaechen koennte. Und diese auf alle Weise zu befoerdern, die Religion auch selbst dem Unglaeubigen oder Flatterer hoechst feyerlich und heilig zu machen, ist ein fuer allemal die erste und groeßte Pflicht der religioesen Musick und Poesie. ${ }^{55}$

Martha. (eilt auf sie zu.)

Ich hab' ihn gesehn - den heiligen Gott,

Erwache, Maria, er kommt!

Ich flog dem heiligen entgegen,

So stuerzte meine Traene - so stuerzt' ich

$\mathrm{Zu}$ seinen Fuessen, faßte seine Kniee:

$53 \mathrm{Zu}$ Niemeyer siehe neben Menne: August Hermann Niemeyer vor allem auch Blanken: Franz Schuberts "Lazarus", S. 103-146.

54 Vgl. Blanken: Franz Schuberts "Lazarus«, S. 114f. u. 121f.

55 Niemeyer: Ueber das religioese Drama, S. 57. Zum im 18. Jahrhundert sowohl theologisch als auch musikästhetisch sowie poetologisch stark diskutierten Problem, ob und in welchem Maße alttestamentarische Sujets auf die Bühne und in die Kirche gebracht werden können und den damit verbundenen christologischen Deutungen des AT (bspw. Adam als Protojesus oder Isaaks Opferung als typologisches pre-enactment des Opfertods Christi) siehe Heidrich: Die protestantische Kirchenmusikanschauung im 18. Jahrhundert, S. 192f. u. 198. Zu den damit zusammenhängenden Problemen von `Orientalismus` und einer (antisemitisch angehauchten) Abgrenzung zum Judentum vgl. Hilliard: Klopstock und das Alte Testament. 
»Waerst $\mathrm{Du}$, ach waerst Du hier gewesen «

"Mein Bruder waere nicht gestorben, "

»Doch noch giebt dir dein Gott was du begehrst!«

Da lächelt er auf mich herab und sprach:

»Dein Bruder, Martha, soll vom Tod erstehn ${ }^{56}$

Während es für Niemeyer kein ausreichender Grund ist, Jesus als Sänger auf die Bühne zu bringen, um ihn mithilfe der Musik vor der Profanierung durch die Bühne zu retten, ist es bei Herder genau die Musik, die es ihm erlaubt, Jesus dramatisch und bühnenmäßig darstellungsfähig zu machen. Bei Herder kommt Jesus auf die Bühne, nicht nur, weil er ihn im Rahmen seiner anthropologischen Religionsphilosophie als Menschen sieht, sondern auch, weil es die Musik ist, die die Exorbitanz der Offenbarung und die Exorbitanz der Wunder kommunizierbar und darstellbar macht. Es ist für Herder entscheidend, dass Jesus singt und nicht einfach redet. In Herders opernförmigem Oratorium konvergieren somit Gattungstheorie, Gattungsgeschichte, Theologie, Poetologie und Musikästhetik. Das heißt, dass die Musikästhetik in die Theologie interveniert und vice versa. Diese Konvergenz erlaubt es Herder auch, dermaßen stark in die biblische Wundergeschichte einzugreifen und Lazarus im Todeskuss mit Maria ein zweites Mal - und dieses Mal zum ewigen Leben - sterben zu lassen. Es ist die musikalisch-akustische Form des Oratoriums, die die Wundergeschichte poetisch darstellbar macht, weil damit im Gegensatz zu Niemeyers sachlich-logischer Erklärung des Wunders das Wunder ontologisch in der Schwebe bleibt. Es ist für Herders Text nicht maßgeblich, ob die Auferweckung des Lazarus Wahrheitsgehalt hat, vielmehr ist entscheidend, dass Jesus als Sänger und Mensch bzw. genauer als singender Mensch und singender Gott darstellungsfähig wird. Die dramatische `énonciation` wird qua Singen, d.h. qua Musik, vor der Profanierung durch die Bühne gefeit. Gleichwohl bleibt dieses herdersche Unternehmen im Hinblick auf die hybride opernhaftoratorienhafte Form des kleinen Textes äußerst prekär. Jesus' Stellung inmitten der Zusammenführung von weltlicher Oper und geistlichem Oratorium ist ambivalent und theologisch keineswegs stringent. Diese Ambivalenz fügt sich in die musikästhetische und theologische Diskurslandschaft des 18. Jahrhunderts ein, da wir hier eine kontroverse Diskussion

Niemeyer: Lazarus, oder die Feyer der Auferstehung, S. 142f. Siehe für weitere Beispiele indirekter Rede auch dort S. 147ff. - Zu Niemeyers >religiösem Drama aus musikwissenschaftlicher Sicht siehe neben den erwähnten Arbeiten von Blanken, Heidrich/Lütteken: Das Monologische als Denkform in der Musikzwischen 1760 und 1785, S. 386-404 auch Waczkat: Niemeyers Abhandlung Ueber das religioese Drama. - Zur Problematik der Profanierung des heiligen biblischen Stoffes auf der weltlichen Bühne und damit zum Verbot des biblischen Dramas in Form der Zensur (im Österreich des 19. Jahrhunderts) vgl. Blanken: Franz Schuberts »Lazarus«, S. 84-97. 
über verschiedene Aspekte der Oratoriumstheorie haben, unter anderem: Welche Sujets dürfen in einem Libretto verarbeitet werden (Altes und/oder Neues Testament)? Darf Jesus Christus auf der Bühne als Figur erscheinen? Darf er singen? Darf er sprechen? In welchem Verhältnis stehen »opernhaft

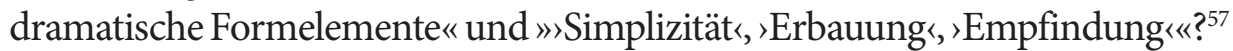
Welche Funktion hat der Chor? In diesem Kontext kommen zwei miteinander eng korrelierende Unterscheidungen zum Einsatz: Erstens die zwischen gottesdienstlicher und außergottesdienstlicher Kirchenmusik, ${ }^{58}$ wobei der Chor in seiner volksbildenden Funktion als zwingend notwendig für die gottesdienstliche Form angesehen wird und zweitens die - insbesondere von Niemeyer eingezogene Unterscheidung - zwischen kirchlich und religiös, was dann zu der gattungsspezifischen Differenz Kirchenmusik/religiöse Dichtung ausgeprägt wird. Religiöse Dichtung, wie sie Niemeyer schreibt, ist prinzipiell nicht für den Gottesdienst bestimmt, was dem Dichter erlaubt, sich von den expliziten heteronomen Vereinnahmungen durch theologisch-religiöse Vorgaben zugunsten poetisch-künstlerischer Freiheiten zu emanzipieren. ${ }^{59}$

\section{4. »Die Basis der heiligen Musik ist Chor"}

Es ist höchst interessant, was Johann Christoph Friedrich Bach (1732-95) in seiner Vertonung des herderschen Librettos ändert (1773). Während Herder nach dem ins ewige Leben führenden Todeskuss von Lazarus und Maria den Text mit Chorälen ausklingen lässt, gleichsam diesen zweiten Tod als ewiges Leben jubilierend feiert, schreibt Bach die Schlusschoräle in ein Tenorsolo um:

Heidrich: Die protestantische Kirchenmusikanschauung im 18. Jahrhundert, S. 184. Zur kontroversen Debatte um Oratorium und Libretto um 1780 siehe ebd. sehr anschaulich S. 184-230.

58 Vgl. ebd., S. 162.

59 S. hierzu prägnant ebd., S. 196: »Die fraglichen Texte sind indes durch drei wichtige Eigenschaften geprägt, die dem Genre >religiöses Drama‘, somit einem gewichtigen Bereich des zeitgenössischen Oratoriumschaffens, einen eigenständigen Platz in der Kirchenmusikdiskussion zuweisen: Sie sind (1.) prinzipiell nicht für die Kirche bestimmt, sie eignen sich (2.) vor allem für die Gebildeten, weshalb (3.) die `Nothwendigkeit der durchgängige[n] Simplicität‘ entfällt. [...] Niemeyers Anschauung zeichnet sich durch eine im sonstigen Verständnis nicht immer selbstverständliche Differenzierung der Begriffe `kirchlich und `religiös $`$ aus: Kirchenmusik ist im eingeschränkten Sinne gottesdienstliche, in der Kirche tatsächlich zu verwendende Musik. Sie muß mit Rücksicht auf das Volk simpel sein, ohne eigentlichen poetischen Anspruch [...]. >Religiöse Dichtung und Musik stehen dagegen außerhalb gottesdienstlicher Funktionalität, sie sind nicht für den `Hauffen`, und somit fordert Niemeyer eine größere poetisch-artifizielle Freiheit des Dichters«. - Lütteken argumentiert, dass Niemeyer drei Gattungen differenziert: Lied, Kantate (und synonym Oratorium) und Drama (= religiöses Drama) (Lütteken: Das Monologische als Denkform in der Musik zwischen 1760 und 1785, S. 394). 


\section{Herder:}

Maria.

Wir wandeln Hand in Hand durchs Leben -

Lazarus.

Im Todeskuß zum schönern Leben -

Beide.

Gen Himmel hin, die schöne Bahn.

O Freund, nimm unsre Thränen an!

Choräle.

(Alte Kirchenmelodie: Jesus Christus, unser Heiland etc.)

1.

Auferstehung Gottes, Du wirst sein!

Mit Jesu gehn wir ein

Ins Himmelsleben.

O Tod, wo ist Dein Beben?

Wo wird es sein?

Chor.

Der Tod verschlungen in Sieg,

Tod, wo ist Dein Pfeil? Hölle, Dein Sieg?

2.

Auferstehung Gottes, Du wirst sein!

Kein Pilger wallt allein,

Sind Alle Brüder,

Und Brüder Jesu! Glieder

Der Krone sein!

Chor.

In der Auferstehung Gottes die Gerechten werden sein Wie Engel Gottes im Himmel!

3.

Auferstehung Gottes, Du wirst sein,

Nicht Schicksal mehr wird sein!

Sind überwunden

Der Trennung bange Stunden,

Der Erde Pein!

Chor.

Meine Seele sterbe

Des Todes der Gerechten!

Mein Ende sei

Ihr Ende! $!^{60}$

60 Herder: Die Auferweckung des Lazarus, S. 517f. 
Bach

Solo.

109
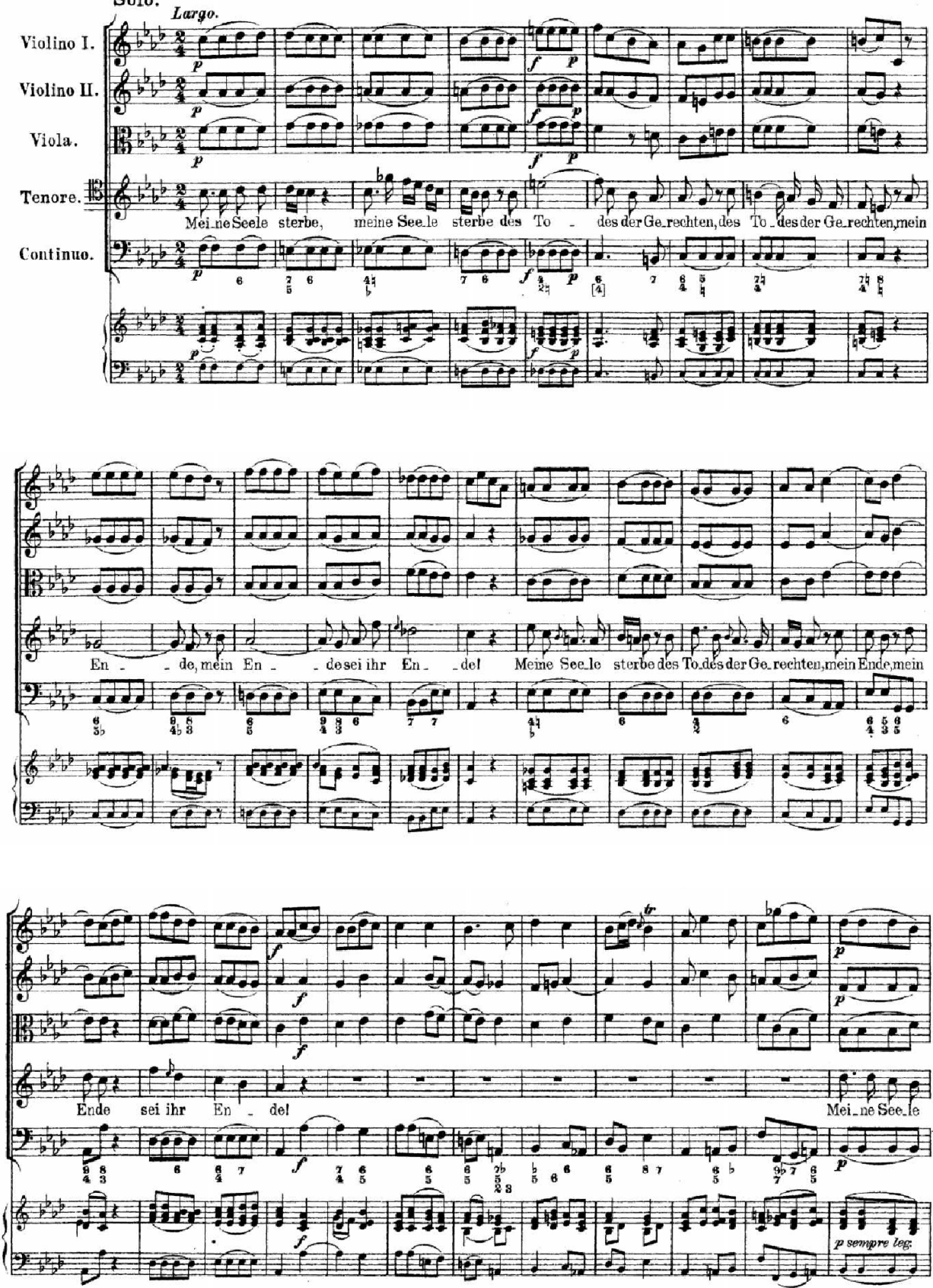

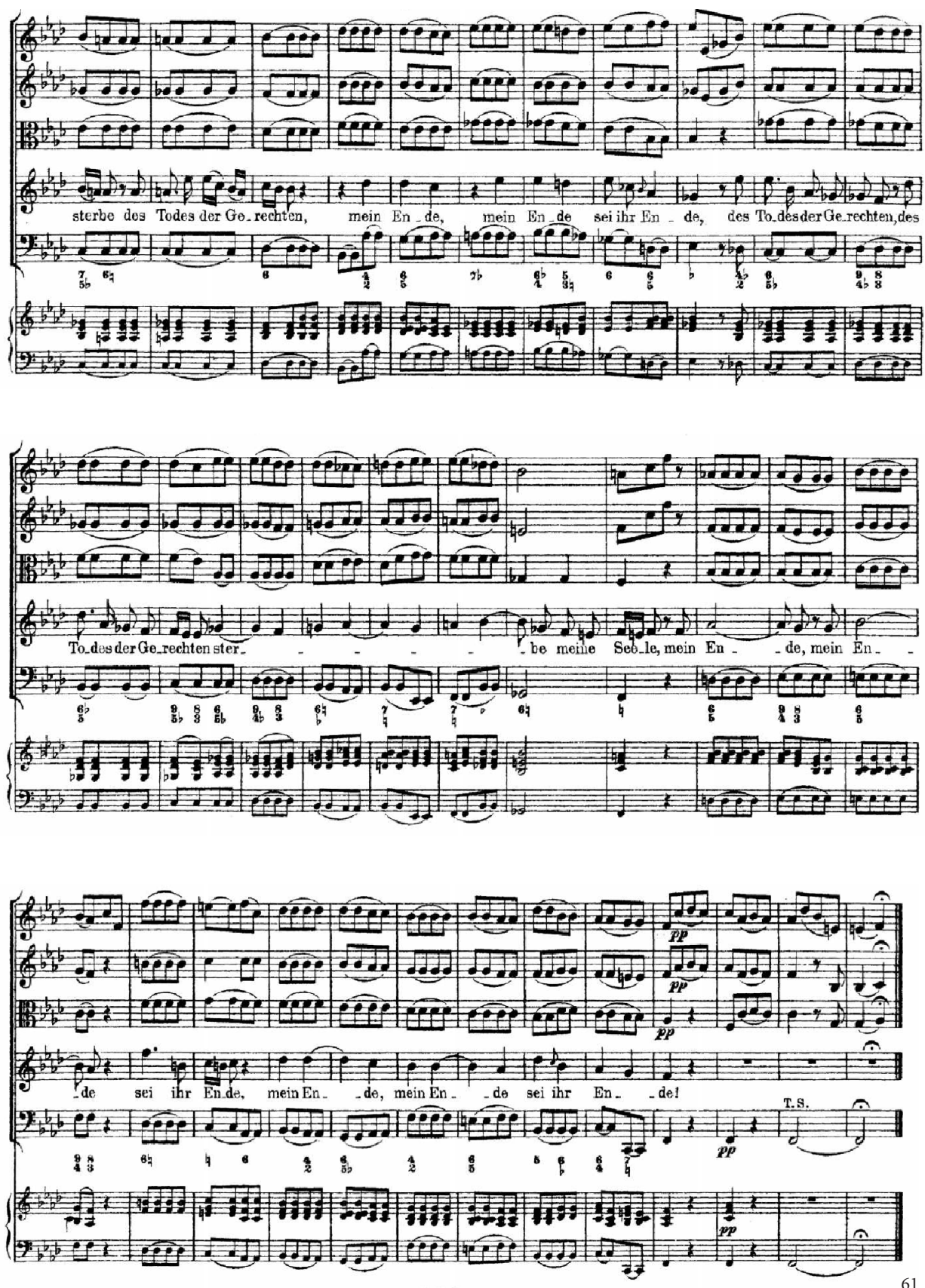

61 Bach: Die Auferweckung des Lazarus, S. 109f. - Einer weiteren Studie, die extrem ungesicherte Editionslage beachtend, mag vorbehalten sein, den alternativen Schluss der Auferweckung des Lazarus in Sämtliche Werke, Band XXVIII, S. 44 zu analysieren. Hier endet es auch mit dem Chor (»Meine Seele sterbe/ Des Todes der Gerechten!/ Mein Ende sei/ Ihr Ende!«), aber kurz 
Es verwundert nicht, dass Herder gute 20 Jahre nach seinem Lazarus in seiner theologisch-musiktheoretischen Schrift Caecilia (1793) die Liturgie, das Lobpreisen, die Andacht und die Begeisterung kurzschließt und dabei den Chor als liturgisch zentral betrachtet:

Die Basis der heiligen Musik ist Chor: denn eine Gemeine soll singen, und wenn zwei oder drei versammelt wären, so machen sie mit der ganzen Christenheit auf Erden Eine Gemeine. Arien also, Duette, Terzette u. dgl. sind nie das Hauptwerk einer Musik der Kirche, gesetzt, daß sie auch in die Kirche gehörten. Nur auf dem Wege des Chors, (im weitesten Verstande genommen,) gelangt man zu jener Bewegung und Rührung, die diese Musik erfodert. Die tiefste Demuth, ja ich möchte sagen, Vernichtung und Zerschmelzung vor Gott, alle Ermunterungen zu Trost, Hoffnung und Freude, jene Ausbrüche des Glaubens, der Hoffnung, Frage und Antwort, Zweifel und Zuversicht, Kummer und Trost, Fluch und Segen sind in den reichen Sätzen und Gegensätzen der Chorsprache alten und neuen Testaments zu finden. ${ }^{62}$

Der Chor ist das entscheidende Moment der Erfahrbarkeit der ambivalenten Erfahrbarkeit von Offenbarung und Wunder. So ist es nur konsequent, dass Herders Lazarus mit einem Chor endet. Gleichwohl ist zu betonen, dass Herder 1793 zwar nicht explizit, aber doch deutlich seinen Lazarus als außergottesdienstlichen, nichtliturgischen Text einordnet. Wie wir sehen konnten, ist sein Lazarus zum Teil stark erzählerisch und dramatisch-dialogisch und damit opernhaft strukturiert. Für solche Texte gilt aber, dass sie nicht für die Kirche gedacht sein können:

Hiemit zeigt sich also, daß die Kirchenmusik auf keine Weise dramatisch seyn könne, und wenn sie dies seyn wolle, sie ganz ihren Zweck verfehle. Auf dem Theater ist alles auf dramatische Vorstellung, Charakterschilderung, aufs Spiel der Personen eingerichtet; hier zeigen sich, wie gesagt, keine Personen, hier wird nichts repräsentiret. Es sind reine, unsichtbare Stimmen, die unmittelbar mit unserm Geist und Herzen reden. Wollte man biblische Geschichten dramatisiren; so gehören sie nicht für die Kirche, sondern mögen zu Hause in sogenannten geistlichen Cantaten gesungen oder gespielt werden. ${ }^{63}$

Damit kennzeichnet Herder seinen Lazarus im Nachhinein als 'geistliche Cantater. Dies ändert aber nichts daran, dass der Chor das entscheidende anhermeneutische Moment bleibt und sowohl in der Kirche als auch außerhalb der Kirche die Erfahrbarkeit der ambivalenten Erfahrbarkeit von Offenbarung und Wunder am klarsten, eindrücklichsten und intensivsten verkörpert.

vorher werden diese Zeilen auch von einer Solostimme gesungen. Es mag sein, dass Bach sich auf diese Version bezieht und deshalb die Solostimme einsetzt, es erklärt aber nicht, wieso er das Oratorium - im Gegensatz zu beiden herderschen Fassungen - nicht mit dem Chorgesang enden lässt. 


\section{Literaturverzeichnis}

\section{Quellen}

Bach, Johann Christoph Friedrich: Die Auferweckung des Lazarus [1773]. In: Die Kindheit Jesu (1773). Die Auferweckung des Lazarus (1773). Hg. Georg Schünemann. Leipzig: Breitkopf \& Härtel 1917, S. 40-110.

Herder, Johann Gottfried: Briefe, das Studium der Theologie betreffend [1780/81]. In: ders.: Werke in zehn Bänden. Bd. 9/1: Theologische Schriften. Hg. G. Arnold. Frankfurt/M.: Deutscher Klassikerverlag 1994, S. 139-607.

Herder, Johann Gottfried: Vom Erlöser der Menschen. Nach unsern drei ersten Evangelien (1796). In: ders.: Werke in zehn Bänden. Bd. 9/1: Theologische Schriften. Hg. G. Arnold. Frankfurt/M.: Deutscher Klassikerverlag 1994, S. 609-724.

Herder, Johann Gottfried: Viertes Kritisches Wäldchen [1769] In: ders.: Werke in zehn Bänden. Bd. 2: Schriften zur Ästhetik und Literatur 1767-1781. Hgg. G. Arnold [u.a.]. Frankfurt/M.: Deutscher Klassikerverlag 1993, S. 247-442.

Herder, Johann Gottfried: Sämtliche Werke. Bd. XXVIII. Hgg. Bernhard Suphan, Carl Redlich. Berlin: Weidmannsche Buchhandlung 1877-1913 (zweiter Nachdruck als Sämtliche Werke. Hildesheim [u.a.]: Olms 1994f.).

Herder, Johann Gottfried: Sämtliche Werke. Bd. XXXIII. Hgg. Bernhard Suphan, Carl Redlich. Berlin: Weidmannsche Buchhandlung 1877-1913 (zweiter Nachdruck als Sämtliche Werke. Hildesheim [u.a.]: Olms 1968).

Herder, Johann Gottfried: Christliche Schriften. In: ders.: Sämtliche Werke. Bd. XIX. Hgg. Bernhard Suphan, Carl Redlich, Reinhold Steig. Berlin: Weidmannsche Buchhandlung 1877-1913 (dritter Nachdruck als Sämtliche Werke. Hildesheim [u.a.]: Olms 1994f.).

Herder, Johann Gottfried: Die Auferweckung des Lazarus. Eine biblische Geschichte zur Musik [1772]. In: ders: Werke. Erster Theil. Gedichte. Hg. Heinrich Dünker. Berlin: Gustav Hempel 1879, S. 514-519.

Herder, Johann Gottfried: Cäcilia. In: ders.: Zerstreute Blätter (Fünfte Samlung). Gotha: Carl Wilhelm Ettinger 1793, S. 287-320.

Klopstock, Friedrich Gottlieb: Biblische Dramen. In: ders.: Werke und Briefe. Historischkritische Ausgabe. Abt. Werke: V. Hgg. Horst Gronemeyer [u.a.]. Berlin: de Gruyter 2005.

Niemeyer, August Hermann: Ueber das religioese Dama so fern es für die Musick bestimmt ist [1778]. In: ders.: Gedichte. Carlsruhe: Christian Gottlieb Schmieder 1783, S. 47-64.

Niemeyer, August Hermann: Lazarus, oder die Feyer der Auferstehung f1778]. In: ders.: Gedichte. Carlsruhe: Christian Gottlieb Schmieder 1783, S. 117-159.

Reimarus, Hermann Samuel: Apologie oder Schutzschrift für die vernünftigen Verehrer Gottes [1735-1767/68]. Hg. Gerhard Alexander. Frankfurt/M.: Insel 1972.

Semler, Johann Salomo: Abhandlung von freier Untersuchung des Canon [1771-75]. Gütersloh: Gütersloher Verlagshaus 1967.

\section{Sekundärliteratur}

Alkier, Stefan: Jenseits von Entmythologisierung und Rehistorisierung - Skizzen zu einer Semiotik des Wunderbaren im frühen Christentum. In: Religion zeigen. Religionspädagogik und Semiotik. Hgg. Bernhard Dressler, Michael Meyer-Blanck. Münster: LIT 1998, S. 27-60. 
Alkier, Stefan: Wunder und Wirklichkeit in den Briefen des Apostels Paulus. Ein Beitrag zu einem Wunderverständnis jenseits von Entmythologisierung. Tübingen: Mohr Siebeck 2001.

Alkier, Stefan: Wen wundert was? Einblicke in die Wunderauslegung von der Aufklärung bis zur Gegenwart. »ZNT« 7 (2001), S. 2-5.

Arnold, Günter: Von den letzten Dingen - eschatologische Elemente in Herders Werk und ihre Quellen. In: Johann Gottfried Herder. Aspekte seines Lebenswerkes (Arbeiten zur Kirchengeschichte 92). Hgg. Martin Keßler, Volker Leppin. Berlin, New York: de Gruyter, S. 383-411.

Berger, Christian (Hg.): Musik jenseits der Sprache (Freiburger Beiträge zur Musikwissenschaft 6). Freiburg: Rombach 2004.

Blanken, Christine: Franz Schuberts »Lazarus « und das Wiener Oratorium zu Beginn des 19. Jahrhunderts (Schubert: Perspektiven - Studien 1). Stuttgart: Franz Steiner 2002.

Bultmann, Christoph; Markus Buntfuß, Claas Cordemann, Martin Keßler: II.2 Theologie. In: Herder Handbuch. Hgg. Stefan Greif, Marion Heinz, Heinrich Clairmont. München: Fink 2016, S. 319-385.

Cordemann, Claas: Herders christlicher Monismus. Eine Studie zur Grundlegung von Johann Gottfried Herders Christologie und Humanitätsideal (Beiträge zur historischen Theologie 154). Tübingen: Mohr Siebeck 2010.

Geppert, Alexander, Till Kössler: Einleitung: Wunder der Zeitgeschichte. In Wunder. Politik und Poetik des Staunens im 20. Jahrhundert. Hgg. Alexander Geppert, Till Kössler. Berlin: Suhrkamp 2011, S. 9-68.

Gess, Nicola: Staunen als ästhetische Emotion. Zu einer Affektpoetik des Wunderbaren. In: Wie gebannt. Ästhetische Verfahren der affektiven Bindung von Aufmerksamkeit. Hgg. Martin Baisch, Andreas Degen, Jana Lüdtke. Freiburg: Rombach 2013, S. 115-132.

Greif, Stefan, Marion Heinz, Heinrich Clairmont (Hgg.): Herder Handbuch. München: Fink 2016.

Grizelj, Mario: Wunder und Wunden. Religion als Formproblem von Literatur (Klopstock - Kleist - Brentano). München: Fink 2018.

Hajduk, Stefan: Poetologie der Stimmung. Ein ästhetisches Phänomen der frühen Goethezeit. Bielefeld: transcript 2016.

Heidrich, Jürgen: Die protestantische Kirchenmusikanschauung im 18. Jahrhundert. Studien zur Ideengeschichte >wahrer Musik (Abhandlungen zur Musikgeschichte 7). Göttingen: Vandenhoeck \& Ruprecht 2001.

Hilliard, Kevin: Klopstock und das Alte Testament. In: Wort und Schrift - Das Werk Friedrich Gottlieb Klopstocks. Hgg. Kevin Hilliard, Katrin Kohl. Tübingen: Niemeyer 2008, S. 41-60.

Köhler, Rafael: Natur und Form. Energetische Form in der Musiktheorie. Stuttgart: Franz Steiner 1995.

Lütteken, Laurenz: Das Monologische als Denkform in der Musik zwischen 1760 und 1785. Tübingen: Niemeyer 1998.

Matuschek, Stefan: Über das Staunen. Eine ideengeschichtliche Analyse. Tübingen: Niemeyer 1991.

Menne, Karl: August Hermann Niemeyer. Sein Leben und sein Wirken. 2. Aufl. Tübingen: Niemeyer 1995.

Previšić, Boris: Akustische Paradigmen vor der Philologie: Herder. In: Vor der Theorie. Immersion - Materialität - Intensität. Hgg. Mario Grizelj, Oliver Jahraus, Tanja Prokić. Würzburg: Königshausen \& Neumann 2014. S. 337-350. 
Schawelka, Karl: Kunst und Wunder. In: Wunder über Wunder - Wunderbares und Wunderliches im Glauben, in der Natur und in der Kunst. Hg. Kai Uwe Schierz. Bielefeld: Kerber 2007, S. 27-44.

Schneider, Joh. Nikolaus: Ins Ohr geschrieben. Lyrik als akustische Kunst zwischen 1750 und 1800 (Das achtzehnte Jahrhundert Supplemata 9). Göttingen: Wallstein 2004.

Teuber, Bernhard: Sichtbare Wundmale und unsichtbare Durchbohrung. Die leibhafte Nachfolge Christi als Paradigma des anhermeneutischen Schreibens. In: Stigmata. Poetiken der Körperinschrift. Hgg. Bettine Menke, Barbara Vinken. München: Fink 2004, S. 155-179.

Teuber, Bernhard: Die mystische Mär. Eine postmoderne Relecture der mystischen Tradition nach Michel de Certeau. In: Die Kirchenkritik der Mystiker. Prophetie aus Gotteserfahrung. Bd. II: Frühe Neuzeit. Hgg. Mariano Delgado, Gotthard Fuchs. Freiburg: Kohlhammer 2005, S. 225-240.

Waczkat, Andreas: Niemeyers Abhandlung >Ueber das religioese Dama so fern es für die Musick bestimmt ist und die musikdramatischen Reformversuche in der zweiten Hälfte des 18. Jahrhunderts. In: ‘Seyd nicht träge in dem was ihr thun sollt. A August Hermann Niemeyer (1754-1828): Erneuerung durch Erfahrung. Hg. Christian Soboth. Tübingen: Niemeyer 2007, S. 175-184.

Weidner, Daniel: Un-Möglichkeit der Ausnahme. Die Wunderkritik der Aufklärung. In: Formen des Nichtwissens der Aufklärung (Laboratorium Aufklärung, Bd. 4). Hgg. Hans Adler, Rainer Godel. München: Fink 2010, S. 121-140.

Weidner, Daniel: Lesen, Schreiben, Denken - Herders >Leben Jesuk. In: Der frühe und der späte Herder. Kontinuität und/oder Korrektur. Hgg. Gerhard Sauder, Sabine Groß. Heidelberg: Synchron 2007, S. 185-197.

Weidner, Daniel: >Bibeldichtung und dichterische Darstellung. Kain in der Literatur um 1800: Klopstock, Gessner, Coleridge, Byron. »arcadia« 43.2 (2008), S. 299-331.

Welck, Christian: Erzählte Zeichen. Die Wundergeschichten des Johannesevangeliums literarisch untersucht. Mit einem Ausblick auf Joh 21. Tübingen: Mohr Siebeck 1994.

Zimmermann, Ruben: Wundern über >des Glaubens liebstes Kind . Die hermeneutische (De-) Konstruktion der Wunder Jesu in der Bibelauslegung des 20. Jahrhunderts. In: Wunder. Poetik und Politik des Staunens im 20. Jahrhundert. Hgg. Alexander C.T Geppert, Till Kössler. Frankfurt/M.: Suhrkamp, S. 95-125.

Zimmermann, Ruben: Grundfragen zu den frühchristlichen Wundererzählungen. In: Kompendium derfrühchristlichen Wundererzählungen. Bd. 1.: Die Wunder Jesu. Hg. Ruben Zimmermann. Gütersloh: Gütersloher Verlagshaus 2013, S. 7-49. 
